\title{
Monitoreo de la ictiofauna usando grupos taxonómicos superiores en el Parque Nacional Cabo Pulmo, México
}

\author{
Romeo Saldívar-Lucio ${ }^{1,2}$ \& Héctor Reyes-Bonilla ${ }^{1}$ \\ 1. Universidad Autónoma de Baja California Sur. Departamento Académico de Biología Marina. Apartado postal 19-B, \\ CP 23080. La Paz, B.C.S., México; hreyes@uabcs.mx \\ 2. Centro Interdisciplinario de Ciencias Marinas, Instituto Politécnico Nacional. Ave. Instituto Politécnico Nacional s/n. \\ Col. Playa Palo de Santa Rita. Apartado Postal 592, CP 23096. La Paz, B.C.S., México; romeo26_1979@yahoo.com
}

Recibido 18-V-2010. Corregido 09-XII-2010. Aceptado 10-I-2011.

\begin{abstract}
Ichthyofauna monitoring using higher taxa in Cabo Pulmo National Park, México. The uses of biological indicators allow to save time, resources and staff efforts when monitoring programs are conducted. The reef fishes are among the species with biggest potential as indicators since they are conspicuous, easy to identify in the field and have ecological and economical importance. The objective of this study was to evaluate the usefulness of higher taxa to predict fish ecological indices in Cabo Pulmo reef. We generated multiple stepwise regression equations with species abundance data grouped at genera and family levels as factors, and using ecological indices $\left(\mathrm{H}^{\prime}, \mathrm{J}^{\prime}, \Delta^{*}\right)$ as dependent variables. To test the efficiency of the models, the expected indexes were compared with those observed in the field during independent test surveys. Results showed that all models were highly significant, and the best fit occurred at genus level to $\mathrm{H}^{\prime}$, while those used for $\Delta^{*}$, based on family data level, were less precise. The proportion of variables required to apply prediction models of any ecological index never exceeded $41 \%$ of the total taxa (genera or families). In conclusion, the use of regression methods to determine higher taxa indicators is adequate and represents a good potential to be used in monitoring programs. In addition, the use of genera and families as indicators provide logistic benefits as training for monitors is less intense and expensive. Finally, the use of expected-observed comparisons to measure the quality of the evaluations can be useful for managers in order to interpret and communicate results of the scientific endeavors to the society, so they in turn can participate and help to improve and facilitate long-term monitoring at Cabo Pulmo National Park. Rev. Biol. Trop. 59 (2): 871-885. Epub 2011 June 01.
\end{abstract}

Key words: Cabo Pulmo, ecological indices, reef fishes, reef monitoring, marine areas management, taxonomic sufficiency, Gulf of California.

Los arrecifes coralinos son considerados entre los ecosistemas más diversos, complejos y hermosos del planeta (Birkeland 1997), y son utilizados por los seres humanos para: investigación, recreación, fuente de alimentos y actividades económicas (Wilkinson 2002). En el occidente de México, un ejemplo de la relevancia de dichos ecosistemas se encuentra en la localidad de Cabo Pulmo, donde está el arrecife coralino más septentrional del Pacífico oriental y que es considerado el único en su tipo dentro del Mar de Cortés (Arizpe-Covarrubias 2008). La importancia biológica del lugar condujo a su protección oficial como Parque Nacional en 1995 (Diario Oficial de la Federación 1995), lo cual promovió el aumento de visitantes que deseaban desarrollar actividades de poco impacto en el arrecife, como el buceo (Martínez-de la Torre 2008). Este modo de recreación es una industria creciente, que para el año 2007 se estimó, que existían entre 5 y 7 millones de buceadores en el mundo (Mograbi \& Rogerson 2007).

Una amenaza para el estado de conservación del Parque Nacional Cabo Pulmo es el acelerado crecimiento de proyectos turísticos 
en sus inmediaciones, que contemplan la construcción de hoteles, marinas y campos de golf (Gámez 2008). Los impactos de un crecimiento descontrolado pueden ser profundos e irreversibles sobre el valor biológico y socioeconómico del sistema (Tilmant 1987, Gámez 2008), y para evitarlos es importante que se regule mediante un programa de manejo adecuado. Es aquí donde el monitoreo biológico juega un papel importante, ya que permite disponer de información que requiere el proceso de toma de decisiones, además de identificar cambios del sistema en el tiempo (Noss et al. 1997, Wilkinson 2002).

En México, el diseño y ejecución del monitoreo en parques nacionales es responsabilidad de la Secretaria de Medio Ambiente y Recursos Naturales a través de la Comisión Nacional de Áreas Naturales Protegidas (LEGEEPA 1988), pero las agencias de gobierno requieren apoyo para ejecutar esta labor, ya que existen limitantes de financiamiento y personal. Este problema es frecuente a nivel mundial (McNally \& Fleishman 2004) y debido a ello se ha popularizado el uso de indicadores biológicos, pues aunque se requiere de personas entrenadas para la identificación de especies, el procedimiento permite optimizar costos y personal (AlmadaVillela et al. 2003).

El presente estudio tuvo como objetivo el reconocer y evaluar el potencial de los grupos mayores de peces (género y familia) del arrecife coralino de Cabo Pulmo como bioindicadores de la estructura de la comunidad íctica a partir de criterios estadísticos. Los resultados muestran que se puede predecir eficientemente la diversidad ecológica y taxonómica de la ictiofauna a partir de datos de abundancia de ciertos grupos indicadores (géneros o familias), lo cual abre la puerta para el diseño y establecimiento de un programa de monitoreo biológico para dar seguimiento a los cambios en la comunidad de peces a largo plazo que respalde el manejo y conservación de este arrecife coralino.

\section{MATERIALES Y MÉTODOS}

El arrecife coralino de Cabo Pulmo se encuentra entre las coordenadas $23^{\circ} 26^{\prime} 00^{\prime \prime} \mathrm{N}$ $-109^{\circ} 25^{\prime} 00^{\prime}$ W y y está conformado por franjas de roca y coral que se extienden hasta los $15 \mathrm{~m}$ de profundidad que se orientan en dirección noreste-suroeste. El arrecife se encuentra dentro del Parque Nacional Cabo Pulmo, cuya superficie es de 7 111ha, un 99\% es la porción marina (DOF 1995). Según Álvarez-Filip et al. (2006) y Riegl et al. (2007), el piso del arrecife esta conformado en mas del $40 \%$ por roca basáltica, aproximadamente 10 a $20 \%$ de coral muerto y una cobertura del $21 \%$ de coral vivo que se concentra hacia el centro del arrecife y es dominado por Pocillopora verrucosa (Ellis \& Solander, 1786). La elevada abundancia de coral muerto que se observa en Cabo Pulmo es resultado del blanqueamiento producido por el evento de oscilación sureña de El Niño en 1997 y una serie de ciclones que impactaron el área entre 2001 y 2003 (Álvarez-Filip et al. 2006). No obstante, desde hace décadas se sabe que la compleja estructura física de este arrecife favorece la abundancia y riqueza de peces (Brusca \& Thomson 1975, Robinson \& Thomson 1992); en la zona se han registrado casi 250 especies, varias endémicas del Golfo de California (Villareal-Cavazos et al. 2000).

La zona de Cabo Pulmo tuvo pesca artesanal por décadas, lo cual llegó a representar una amenaza para las poblaciones de peces (Arizpe-Covarrubias 2004). Sin embargo, la población local dejó de capturarlos a partir de la declaratoria del parque (DOF 1995), por lo que a la fecha la comunidad íctica muestra señales evidentes de recuperación. Actualmente, se sabe de la presencia de grandes cardúmenes y de un notable aumento en la talla de peces de importancia comercial (Álvarez-Filip \& Reyes-Bonilla 2006, CONANP 2006). Ello ha provocado que estos organismos sean en gran medida los responsables del alto valor que tiene el arrecife como sitio de recreación (ReyesBonilla \& Beltrán-Ramírez 1998, VillarrealCavazos et al. 2000).

Para este estudio se usaron los datos de censos efectuados en Octubre de 2003, Febrero de 2004, Octubre de 2004 y Octubre de 2005. Para realizar los censos se empleó el método del cilindro imaginario que fue propuesto por 
Bohnsack \& Bannerot (1986) y fue modificado por Villarreal-Cavazos et al. (2000). Durante los conteos se observaron aquellos peces que entraron en un cilindro imaginario de $5 \mathrm{~m}$ de radio $\left(\approx 79 \mathrm{~m}^{2}\right.$ de muestreo) por un tiempo de observación de $15 \mathrm{~min}$, donde los primeros cinco se utilizaron para elaborar una lista de las especies observadas y los diez restantes para registrar sus abundancias. En las visitas de 2003 a 2005 se hicieron 89 cilindros en total y se muestreó un área de $7031 \mathrm{~m}^{2}$ (aproximadamente $1422 \mathrm{~m}^{2}$ por época de muestreo). Con el fin de validar los modelos de predicción que se describen más adelante, en mayo de 2006 se hicieron nuevos registros de la abundancia de peces en el arrecife, para ello se efectuaron 18 cilindros que equivalen a un área de $1422 \mathrm{~m}^{2}$.

Para describir la estructura comunitaria a partir de los datos de abundancia de peces, se estimaron los índices ecológicos de ShannonWiener $\left(H^{\prime} ; \log _{e}\right)$, equidad de Pielou (J'), e índice promedio de Diferenciación Taxonómica $\left(\Delta^{*}\right.$, Clarke \& Warwick 2001). Posteriormente, los valores de abundancia obtenidos de los censos se agruparon a nivel género y a nivel familia y a partir de ahí se llevó a cabo la construcción de modelos de predicción mediante análisis de regresión múltiple, con la rutina por pasos ("stepwise") y la versión "hacia adelante" (Zar 1999). Dicha rutina es un procedimiento automático que fue ejecutado con el paquete STATISTICA (v. 6.1.6), en el cual se van agregando variables una a una y su inclusión o rechazo es controlada por una secuencia de pruebas F y se retuvieron sólo aquellas variables cuya contribución al ajuste del modelo es significativa (Tabachnick \& Fidell 1996; Neter et al. 1996). Cada modelo arrojó un subconjunto de taxa que fueron considerados como predictores de las variables de respuesta $\left(\mathrm{H}^{\prime}, \mathrm{J}\right.$ ' y $\left.\Delta^{*}\right)$ y por ende, como los géneros o familias indicadoras.

Finalmente se evaluó la eficiencia de predicción de los modelos con un censo de prueba en mayo de 2006. Con esos nuevos registros de abundancia se calcularon una vez más los índices de diversidad (H', J', $\left.\Delta^{*}\right)$. Paralelamente, se usaron los datos de abundancia de los grupos indicadores previamente seleccionados; se sustituyeron en las ecuaciones de regresión generadas y se hizo la predicción de los valores de todos los índices para cada transecto. Además de tomar en cuenta la significancia y el coeficiente de determinación, en términos operativos consideramos la conveniencia de que el modelo predictivo contemple menos del $60 \%$ de taxa indicadores respecto al total cuantificados por censo. Por último, los valores observados y esperados de diversidad, equidad y diferenciación taxonómica fueron comparados con una prueba t de Student (Zar 1999) y se calculó la diferencia para los promedios de los grupos de datos, misma que se expresó en porcentaje.

\section{RESULTADOS}

Índices ecológicos descriptivos: Durante las cuatro visitas al arrecife de Cabo Pulmo entre los meses de Octubre de 2003 y Octubre de 2005, se registraron un total de 18686 individuos identificados como 69 especies, en 49 géneros y 25 familias. La riqueza de especies entre épocas de muestreo tuvo valores muy similares (Fig. 1), con un promedio general de $16.26 \pm 0.42$, que de acuerdo con el análisis de varianza no presentó diferencias significativas $\left(\mathrm{F}_{3,85}=0.730, \mathrm{p}=0.54\right)$. La abundancia presentó un promedio general de $210 \pm 16$ individuos/ censo, sin diferencias significativas entre épocas de muestreo $\left(\mathrm{F}_{3,85}=1.45, \mathrm{p}=0.234\right)$. En contraste, el índice de Shannon (promedio $1.85 \pm 0.03)$ varió entre épocas $\left(\mathrm{F}_{3,85}=4.23\right.$, $\mathrm{p}=0.008)$; de acuerdo con la prueba a posteriori, H' fue menor en Febrero de 2004 que en Octubre del mismo año (Fig. 1). Para el índice de Equidad (J)', se obtuvo un promedio general de $0.67 \pm 0.01$ y se encontraron diferencias significativas en Febrero de 2004 respecto a los meses de Octubre de 2004 y Octubre de $2005\left(\mathrm{~F}_{3,85}=5.67, \mathrm{p}=0.001\right)$. Finalmente, el índice de diferenciación taxonómica tuvo un promedio general de $72.42 \pm 0.16$, sin diferencias significativas en el tiempo $\left(\mathrm{F}_{3,85}=0.40\right.$, $\mathrm{p}=0.722$ ). En resumen, la comunidad de peces 

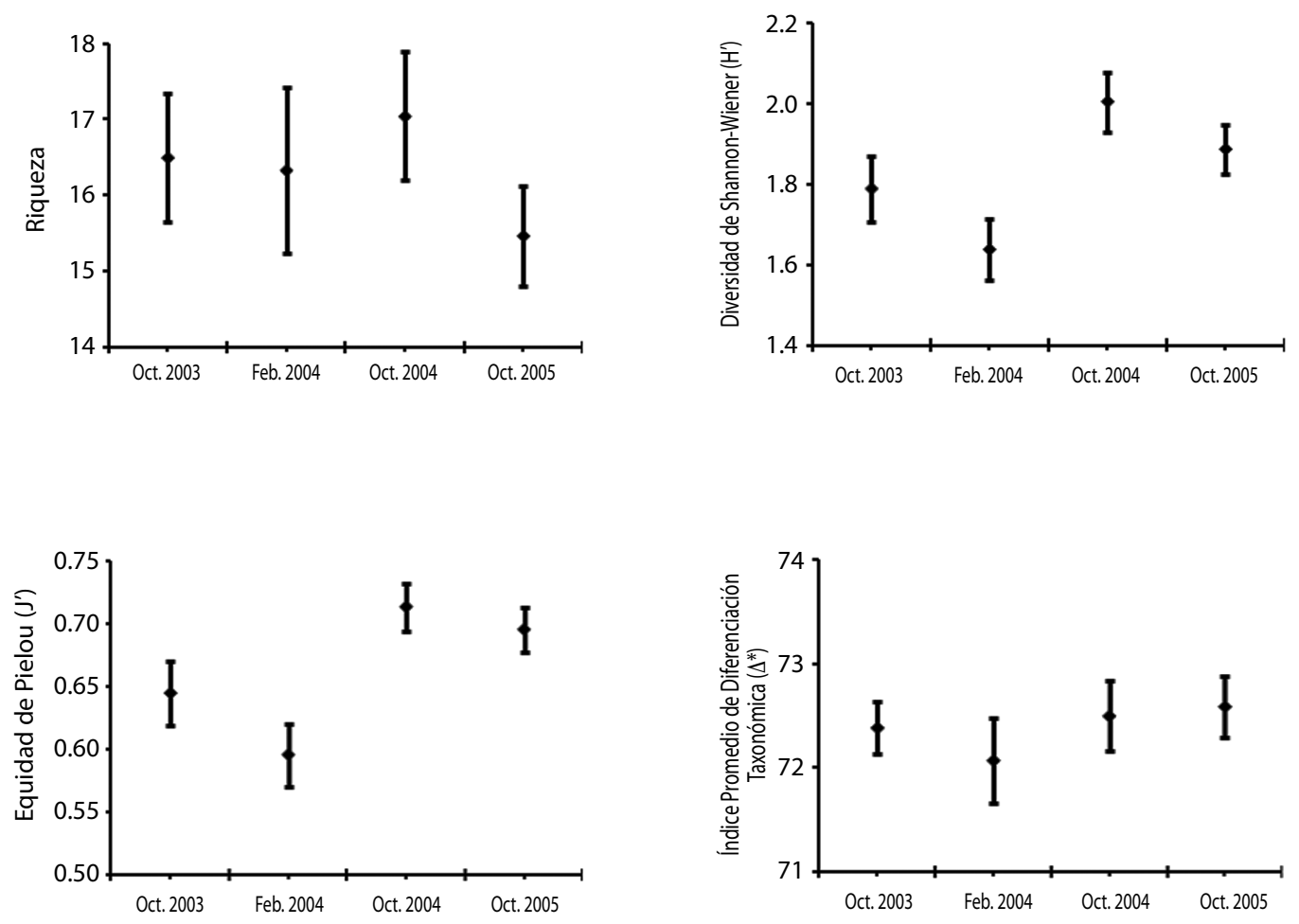

Fig. 1. Índices ecológicos de la comunidad íctica del arrecife de Cabo Pulmo por época de muestreo.

Fig. 1. Ecological índices of the fish community of Cabo Pulmo reef by sampling season.

fue notablemente estable entre 2003 y 2005 , con valores bajos en invierno de 2004 (Fig. 1).

Selección de taxa indicadores mediante análisis de regresión múltiple.

Observaciones generales: Todos los análisis de regresión efectuados en este estudio fueron altamente significativos (Cuadro 1), pero los coeficientes de determinación generalmente fueron menores cuando se utilizaron variables predictoras de mayor jerarquía taxonómica (familia). El coeficiente de determinación más alto $\left(\mathrm{R}^{2}=0.69\right)$ se presentó en la regresión que involucró al índice de diversidad de Shannon-Wiener y la abundancia agrupada a nivel género (Cuadro 1). En contraste, el valor más bajo del coeficiente de determinación
$\left(\mathrm{R}^{2}=0.43\right)$, estuvo en el modelo que relacionó al índice de diferenciación taxonómica y la abundancia agrupada a nivel familia (Cuadro 1).

El número de variables empleadas para la construcción de los modelos fue de 49 a nivel género y 25 a nivel familia, mientras que el número de taxa elegidos por la regresión por pasos fluctuó entre nueve y 20, las cifras más altas siempre correspondieron con las cifras de los coeficientes de determinación mayores (Cuadro 1). El número más bajo de variables seleccionadas para la predicción presentó el $36 \%$ del total de taxa, mientras que el más alto fue de $68 \%$. Se considera que la mayoría de los ajustes fueron eficientes, pues en general se usó menos del $45 \%$ de las variables (Cuadro 1). 
CUADRO 1

Análisis de regresión múltiple por pasos usada para definir taxa indicadores

TABLE 1

Stepwise multiple regressions used to define indicator taxa

\begin{tabular}{ccccc}
$\begin{array}{c}\text { Índice } \\
\text { ecológico }\end{array}$ & $\begin{array}{c}\text { Nivel } \\
\text { taxonómico }\end{array}$ & $\begin{array}{c}\text { Número de variables predictoras } \\
\text { incluidas al inicio del análisis }\end{array}$ & $\begin{array}{c}\text { Número de variables } \\
\text { seleccionadas para la } \\
\text { predicción (\% del total) }\end{array}$ & $\mathrm{R}^{2}$ \\
\hline $\mathrm{H}^{\prime}$ & Género & 49 & $18(37 \%)$ & 0.69 \\
& Familia & 25 & $17(68 \%)$ & 0.60 \\
$\mathrm{~J}$ & Género & 49 & $18(37 \%)$ & 0.65 \\
& Familia & 25 & $9(36 \%)$ & 0.53 \\
$\Delta^{*}$ & Género & 49 & $20(41 \%)$ & 0.54 \\
& Familia & 25 & $15(60 \%)$ & 0.43
\end{tabular}

En todos los casos, $\mathrm{p}<0.0001$. / In all cases, $\mathrm{p}<0.0001$.

\section{Índice de diversidad de Shannon-Wiener (H')}

a) Regresiones a nivel género: $\mathrm{El}$ primer escenario consistió en introducir a la regresión los valores de abundancia agrupados a nivel género. El modelo fue significativo y tuvo un coeficiente de determinación de 0.69 , con 18 géneros escogidos. Destacaron por su relación positiva y significativa con H' los géneros Scarus, Kyphosus, Abudefduf, Bodianus, Paranthias, Cephalopholis y Holacanthus; por otro lado, Thalassoma y Chromis presentaron relaciones negativas y significativas con $\mathrm{H}^{\prime}$. En conjunto, estas nueve variables acumularon el 59\% de la variabilidad explicada (Cuadro 2). Otros dos géneros (ie. Plagiotremus y Gymnothorax) tuvieron relaciones negativas, aunque estas no fueron significativas.

b) Regresiones a nivel familia: Para este nivel de menor resolución taxonómica el modelo fue significativo y tuvo un coeficiente de determinación de 0.60 , con 17 elementos. Entre los predictores de H' destacan las familias Scaridae y Kyphosidae por su fuerte relación positiva y las familias Labridae y Pomacentridae por relacionarse negativa y significativamente con el índice. En conjunto con otras dos variables (Tetraodontidae y Diodontidae), todas esas

\section{CUADRO 2}

Análisis de regresión múltiple por pasos, que relaciona H' con la abundancia a nivel de género

\section{TABLE 2}

Stepwise multiple regression analysis, relating $\mathrm{H}^{\prime}$ and abundance at genus level

\begin{tabular}{|c|c|c|c|c|}
\hline & Beta & $\begin{array}{c}\text { Varianza } \\
\text { explicada } \\
\text { por el factor }\end{array}$ & $\begin{array}{c}\text { Varianza } \\
\text { acumulada }\end{array}$ & $\mathrm{p}$ \\
\hline Intercepto & 1.694 & & & 0.00 \\
\hline Scarus ** & 0.043 & 0.15 & 0.15 & 0.00 \\
\hline Thalassoma $* *$ & -0.003 & 0.16 & 0.31 & 0.00 \\
\hline Kyphosus ** & 0.048 & 0.11 & 0.42 & 0.00 \\
\hline Abudefduf** & 0.021 & 0.05 & 0.47 & 0.00 \\
\hline Bodianus ** & 0.025 & 0.03 & 0.50 & 0.01 \\
\hline Chromis ** & -0.002 & 0.03 & 0.53 & 0.00 \\
\hline Paranthias $* *$ & 0.002 & 0.02 & 0.55 & 0.02 \\
\hline Cephalopholis ** & 0.094 & 0.02 & 0.58 & 0.03 \\
\hline Holacanthus ** & 0.015 & 0.02 & 0.59 & 0.01 \\
\hline Plagiotremus & -0.053 & 0.02 & 0.61 & 0.07 \\
\hline Halichoeres & 0.011 & 0.01 & 0.62 & 0.19 \\
\hline Gymnothorax & -0.128 & 0.02 & 0.64 & 0.07 \\
\hline Mugil & 0.057 & 0.01 & 0.65 & 0.10 \\
\hline Scorpaena & 0.252 & 0.01 & 0.66 & 0.11 \\
\hline Sufflamen & 0.017 & 0.01 & 0.67 & 0.16 \\
\hline Balistes & 0.022 & 0.01 & 0.68 & 0.14 \\
\hline Nicholsina & 0.036 & 0.01 & 0.69 & 0.18 \\
\hline Serranus & 0.041 & 0.01 & 0.69 & 0.24 \\
\hline
\end{tabular}

$\mathrm{R}^{2}=0.69 ; \mathrm{F}_{18,70}=8.74 ; \mathrm{p}<0.00001$. Se denotan con $* *$ las relaciones significativas $(\mathrm{p}<0.05)$.

$\mathrm{R}^{2}=0.69 ; \mathrm{F}_{18,70}=8.74 ; \mathrm{p}<0.00001$. Significant relations are marked with **. 
familias acumularon el $49 \%$ de la variabilidad explicada (Cuadro 3).

\section{Índice de equidad de Pielou (J')}

a) Regresiones a nivel género: $\mathrm{Al}$ agrupar la abundancia a nivel género y relacionar con J' como variable dependiente, se encontró que el modelo fue significativo y el valor del coeficiente de determinación fue 0.65 . Hubo 18 taxa en la regresión y aquellos cuya abundancia estuvo relacionada significativa y positivamente con el índice fueron Kyphosus, Cirrhitichtys y Abudefduf; mientras que Thalassoma, Chromis y Gymnothorax presentaron ajustes inversos. Otros dos géneros (ie. Plagiotremus

\section{CUADRO 3}

Análisis de regresión múltiple por pasos, relacionando H' con la abundancia a nivel de familia

TABLE 3

Stepwise multiple regression analysis, relating $\mathrm{H}^{\prime}$ and abundance at family level

$\begin{array}{lcccc} & \text { Beta } & \begin{array}{c}\text { Varianza } \\ \text { explicada } \\ \text { por el factor }\end{array} & \begin{array}{c}\text { Varianza } \\ \text { acumulada }\end{array} & \mathrm{p} \\ \text { Intercepto } & 1.659 & & & 0.00 \\ \text { Scaridae ** } & 0.046 & 0.18 & 0.18 & 0.00 \\ \text { Labridae ** } & -0.002 & 0.15 & 0.33 & 0.00 \\ \text { Kyphosidae ** } & 0.052 & 0.11 & 0.43 & 0.00 \\ \text { Tetraodontidae } & 0.009 & 0.02 & 0.46 & 0.18 \\ \text { Diodontidae } & 0.035 & 0.02 & 0.48 & 0.30 \\ \text { Pomacentridae ** } & -0.001 & 0.02 & 0.49 & 0.02 \\ \text { Mugilidae } & 0.066 & 0.02 & 0.51 & 0.07 \\ \text { Balistidae } & 0.012 & 0.01 & 0.52 & 0.28 \\ \text { Mullidae } & 0.001 & 0.01 & 0.53 & 0.30 \\ \text { Pomacanthidae } & 0.010 & 0.01 & 0.54 & 0.11 \\ \text { Scorpaenidae } & 0.250 & 0.01 & 0.55 & 0.16 \\ \text { Fistularidae } & 0.059 & 0.01 & 0.56 & 0.20 \\ \text { Haemulidae } & 0.002 & 0.01 & 0.57 & 0.20 \\ \text { Acanthuridae } & 0.004 & 0.01 & 0.58 & 0.15 \\ \text { Chaetodontidae } & 0.009 & 0.01 & 0.58 & 0.18 \\ \text { Ostraciidae } & 0.305 & 0.01 & 0.59 & 0.24 \\ \text { Cirrhitidae } & 0.004 & 0.01 & 0.60 & 0.27\end{array}$

$\mathrm{R}^{2}=0.60 ; \mathrm{F}_{17,71}=6.20 ; \mathrm{P}<0.00001$. Se denotan con $* *$ las relaciones significativas $(\mathrm{p}<0.05)$.

$\mathrm{R}^{2}=0.60 ; \mathrm{F}_{17,71}=6.20 ; \mathrm{P}<0.00001$. Significant relations are marked with $* *$. y Cirrhitus) tuvieron una relación negativa con J', pero no fue significativa (Cuadro 4).

b) Regresiones a nivel familia: En el análisis que consideró la abundancia de especies agrupadas a nivel familia como variables independientes, el modelo fue significativo y el coeficiente de determinación fue de 0.53 . Hubo nueve familias predictoras y las que mostraron una relación positiva y significativa con $\mathrm{J}^{\prime}$ fueron Kyphosidae, Pomacentridae, Scaridae, Cirrhitidae y Mugilidae. La familia Labridae fue la única que tuvo una relación negativa con el índice, y además significativa. En conjunto, esas seis familias explicaron el $51 \%$ de la variabilidad total (Cuadro 5).

\section{CUADRO 4}

Análisis de regresión múltiple por pasos, relacionando J' y la abundancia a nivel de género

\section{TABLE 4}

Stepwise multiple regression analysis, relating $\mathrm{J}^{\prime}$ and abundance at genus level

$\begin{array}{lcccc} & \text { Beta } & \begin{array}{c}\text { Varianza } \\ \text { explicada } \\ \text { por el factor }\end{array} & \begin{array}{c}\text { Varianza } \\ \text { acumulada }\end{array} & \mathrm{p} \\ \text { Intercepto } & 0.676 & & & 0.00 \\ \text { Thalassoma } * * & -0.001 & 0.29 & 0.29 & 0.00 \\ \text { Kyphosus } * * & 0.012 & 0.08 & 0.37 & 0.01 \\ \text { Chromis } * * & -0.001 & 0.05 & 0.42 & 0.00 \\ \text { Scarus } & 0.006 & 0.04 & 0.46 & 0.07 \\ \text { Cirrhitichtys ** } & 0.002 & 0.04 & 0.50 & 0.03 \\ \text { Mugil } & 0.022 & 0.03 & 0.53 & 0.05 \\ \text { Plagiotremus } & -0.019 & 0.03 & 0.55 & 0.05 \\ \text { Abudefduf ** } & 0.004 & 0.01 & 0.57 & 0.01 \\ \text { Gymnothorax } * * & -0.048 & 0.01 & 0.58 & 0.04 \\ \text { Bodianus } & 0.005 & 0.02 & 0.59 & 0.12 \\ \text { Halichoeres } & 0.003 & 0.01 & 0.60 & 0.17 \\ \text { Serranus } & 0.017 & 0.01 & 0.61 & 0.15 \\ \text { Paranthias } & 0.000 & 0.01 & 0.61 & 0.24 \\ \text { Cephalopholis } & 0.018 & 0.01 & 0.62 & 0.21 \\ \text { Cirrhitus } & -0.021 & 0.01 & 0.63 & 0.32 \\ \text { Holacanthus } & 0.002 & 0.01 & 0.63 & 0.20 \\ \text { Acanthurus } & 0.004 & 0.01 & 0.64 & 0.20 \\ \text { Johnrandallia } & 0.002 & 0.01 & 0.65 & 0.31\end{array}$

$\mathrm{R}^{2}=0.65 ; \mathrm{F}_{18,70}=7.07 ; \mathrm{P}<0.00001$. Se denotan con $* *$ las relaciones significativas $(\mathrm{p}<0.05)$.

$\mathrm{R}^{2}=0.65 ; \mathrm{F}_{18,70}=7.07 ; \mathrm{P}<0.00001$. Significant relations are marked with $* *$. 
CUADRO 5

Análisis de regresión múltiple por pasos, relacionando J’ y la abundancia a nivel de familia

TABLE 5

Stepwise multiple regression analysis, relating $\mathrm{J}^{\prime}$ and abundance at family level

$\begin{array}{lcccc} & \text { Beta } & \begin{array}{c}\text { Varianza } \\ \text { explicada } \\ \text { por el factor }\end{array} & \begin{array}{c}\text { Varianza } \\ \text { acumulada }\end{array} & \mathrm{p} \\ \text { Intercepto } & 0.702 & & & 0.00 \\ \text { Labridae ** } & -0.001 & 0.28 & 0.28 & 0.00 \\ \text { Kyphosidae ** } & 0.012 & 0.08 & 0.36 & 0.01 \\ \text { Pomacentridae ** } & 0.000 & 0.04 & 0.40 & 0.00 \\ \text { Scaridae ** } & 0.008 & 0.04 & 0.44 & 0.02 \\ \text { Cirrhitidae ** } & 0.002 & 0.04 & 0.48 & 0.01 \\ \text { Mugilidae ** } & 0.023 & 0.03 & 0.51 & 0.04 \\ \text { Ostraciidae } & 0.084 & 0.01 & 0.52 & 0.29 \\ \text { Chaetodontidae } & 0.003 & 0.01 & 0.52 & 0.20 \\ \text { Balistidae } & 0.003 & 0.01 & 0.53 & 0.30\end{array}$

$\mathrm{R}^{2}=0.53 ; \mathrm{F}_{9,79}=9.94 ; \mathrm{P}<0.00001$. Se denotan con $* *$ las relaciones significativas $(\mathrm{p}<0.05)$.

$\mathrm{R}^{2}=0.53 ; \mathrm{F}_{9,79}=9.94 ; \mathrm{P}<0.00001$. Significant relations are marked with $* *$.

\section{Índice promedio de diferenciación taxonómica $\left(\Delta^{*}\right)$}

a) Regresiones a nivel género: $\mathrm{El}$ modelo que consideró la abundancia agrupada a nivel género fue significativo (con 20 variables seleccionadas) y el coeficiente de determinación fue de 0.54. Los géneros relacionados positiva y significativamente con el índice fueron Lutjanus, Diodon, Myripristis y Balistes, mientras que Halichoeres y Nicholsina se relacionaron negativa y significativamente con $\Delta^{*}$. Otros cinco géneros presentaron relaciones negativas, pero no fueron significativas (Cuadro 6).

b) Regresiones a nivel familia: Respecto al análisis que consideró la abundancia agrupada a nivel familia, se introdujeron 25 variables en el modelo, el cual fue significativo con un coeficiente de determinación de 0.43 , el más bajo del estudio. Hubo 15 familias predictoras y las que presentaron relación positiva y significativa fueron Lutjanidae, Diodontidae,
CUADRO 6

Análisis de regresión múltiple por pasos, relacionando $\Delta^{*}$ y la abundancia a nivel de género

\section{TABLE 6}

Results of stepwise multiple regression analysis, relating $\Delta^{*}$ and abundance at genus level

$\begin{array}{lcccc} & \text { Beta } & \begin{array}{c}\text { Varianza } \\ \text { explicada } \\ \text { por el factor }\end{array} & \begin{array}{c}\text { Varianza } \\ \text { acumulada }\end{array} & \mathrm{p} \\ \text { Intercepto } & 72.019 & & & 0.00 \\ \text { Lutjanus } * * & 0.022 & 0.11 & 0.11 & 0.02 \\ \text { Halichoeres ** } & -0.098 & 0.05 & 0.16 & 0.03 \\ \text { Diodon } * * & 0.357 & 0.05 & 0.21 & 0.03 \\ \text { Stegastes } & -0.020 & 0.05 & 0.26 & 0.13 \\ \text { Mulloidichthys } & 0.010 & 0.03 & 0.29 & 0.17 \\ \text { Cirrhitichtys } & 0.027 & 0.03 & 0.31 & 0.06 \\ \text { Myripristis } * * & 0.643 & 0.03 & 0.34 & 0.02 \\ \text { Nicholsina } * * & -0.337 & 0.03 & 0.37 & 0.02 \\ \text { Fistularia } & 0.344 & 0.02 & 0.39 & 0.11 \\ \text { Paranthias } & 0.007 & 0.02 & 0.41 & 0.09 \\ \text { Balistes } * * & 0.182 & 0.02 & 0.43 & 0.03 \\ \text { Cirrhitus } & -0.543 & 0.02 & 0.45 & 0.12 \\ \text { Prionurus } & 0.024 & 0.02 & 0.47 & 0.07 \\ \text { Thalassoma } & -0.004 & 0.02 & 0.48 & 0.14 \\ \text { Johnrandallia } & 0.050 & 0.01 & 0.49 & 0.12 \\ \text { Caranx } & 0.206 & 0.01 & 0.51 & 0.20 \\ \text { Holacanthus } & 0.044 & 0.01 & 0.52 & 0.15 \\ \text { Epinephelus } & -0.136 & 0.01 & 0.52 & 0.29 \\ \text { Kyphosus } & 0.074 & 0.01 & 0.53 & 0.26 \\ \text { Scarus } & -0.057 & 0.01 & 0.54 & 0.29\end{array}$

$\mathrm{R}^{2}=0.54 ; \mathrm{F}_{20,68}=3.97 ; \mathrm{P}<0.00001$. Se denotan con $* *$ las relaciones significativas $(\mathrm{p}<0.05)$.

$\mathrm{R}^{2}=0.54 ; \mathrm{F}_{20,68}=3.97 ; \mathrm{P}<0.00001$. Significant relations are marked with $* *$.

Serranidae, Holocentridae y Acanthuridae; en contraste Labridae y Scaridae tuvieron relaciones negativas y significativas. Estas familias en conjunto y sumadas a Carangidae y Zanclidae explicaron apenas el 35\% de la variabilidad (Cuadro 7).

Censo de prueba: En mayo del 2006 se realizaron 18 censos visuales $\left(1422 \mathrm{~m}^{2}\right.$ de muestreo), en los que se registraron 3537 individuos identificados en 39 géneros y 21 


\section{CUADRO 7}

Análisis de regresión múltiple por pasos, relacionando $\Delta^{*}$ y la abundancia a nivel de Familia

TABLE 7

Stepwise multiple regression analysis, relating $\Delta^{*}$ and abundance at Family level

$\begin{array}{lcccc} & \text { Beta } & \begin{array}{c}\text { Varianza } \\ \text { explicada } \\ \text { por el factor }\end{array} & \begin{array}{c}\text { Varianza } \\ \text { acumulada }\end{array} & \mathrm{p} \\ \text { Intercepto } & 71.591 & & & 0.00 \\ \text { Lutjanidae }^{* *} & 0.027 & 0.11 & 0.11 & 0.00 \\ \text { Diodontidae ** } & 0.354 & 0.04 & 0.15 & 0.04 \\ \text { Carangidae } & 0.233 & 0.04 & 0.19 & 0.17 \\ \text { Serranidae ** } & 0.010 & 0.03 & 0.22 & 0.01 \\ \text { Labridae } * *^{*} & -0.006 & 0.04 & 0.26 & 0.02 \\ \text { Holocentridae *** } & 0.574 & 0.03 & 0.30 & 0.03 \\ \text { Zanclidae } & 0.347 & 0.02 & 0.32 & 0.31 \\ \text { Acanthuridae ** } & 0.029 & 0.02 & 0.33 & 0.03 \\ \text { Scaridae } * * & -0.113 & 0.02 & 0.35 & 0.05 \\ \text { Pomacanthidae } & 0.045 & 0.02 & 0.37 & 0.15 \\ \text { Chaetodontidae } & 0.061 & 0.02 & 0.38 & 0.07 \\ \text { Blennidae } & 0.105 & 0.02 & 0.40 & 0.24 \\ \text { Cirrhitidae } & 0.022 & 0.01 & 0.41 & 0.16 \\ \text { Scombridae } & 0.059 & 0.01 & 0.42 & 0.24 \\ \text { Fistularidae } & 0.263 & 0.01 & 0.43 & 0.27\end{array}$

$\mathrm{R}^{2}=0.43 ; \mathrm{F}_{15,73}=3.68 ; \mathrm{P}<0.0001$. Se marcan con $* *$ las familias cuya abundancia se relacionó significativamente con $\Delta^{*}$.

$\mathrm{R}^{2}=0.43 ; \mathrm{F}_{15,73}=3.68 ; \mathrm{P}<0.0001$. Significant relations are marked with **. familias y de ahí se calculó H', J' y $\Delta^{*}$. Al aplicar los datos de abundancia de los respectivos géneros y familias en las ecuaciones de regresión múltiple (construidas de modo independiente con datos de años previos), y luego tratar de estimar los índices descriptores de la comunidad, resalta el hecho de que no se presentaron diferencias significativas entre los valores promedio observados y calculados en ninguno de los casos y que sus diferencias fueron menores al $4.5 \%$ del valor de cada índice en cinco de los seis exámenes (Cuadro 8). Es decir, la precisión de los modelos predictivos fue notablemente alta en todos los casos. Los porcentajes más altos de diferencia entre medias se presentaron para estimar el índice de Shannon-Wiener (7.59\% del valor real) y el de Pielou (4.02\%). En cambio, los menores porcentajes de diferencia entre medias se presentaron al analizar $\Delta^{*}$ (Cuadro 8). Las gráficas (Fig. 2) muestran que los modelos se comportaron de manera eficiente, a pesar de que no son tan precisos en ciertos casos particulares (transectos). También se puede apreciar cierta tendencia hacia la sobreestimación de $\mathrm{H}^{\prime}$ y $\mathrm{J}^{\prime}$, mientras que los modelos para predecir $\Delta^{*}$ tienden a subestimar. Finalmente, en la predicción de $\Delta^{*}$ mediante la utilización de géneros, se puede apreciar que el ajuste del modelo es muy bueno, excepto por dos puntos que se destacan por su mal ajuste al haber sido gravemente subestimados. No

CUADRO 8

Comparación de los valores de los índices ecológicos observados en el campo y calculados con las ecuaciones de regresión múltiple

TABLE 8

Ecological indices observed in the field, and those calculated with the multiple regression equations

$\begin{array}{ccccccc}\text { Indices } & \begin{array}{c}\text { Taxón } \\ \text { indicador }\end{array} & \text { Promedio observado } & \text { Promedio calculado } & \begin{array}{c}\text { Diferencia } \\ \text { de medias }(\%)\end{array} & \text { t } & \mathrm{p} \\ \text { H' } & \text { Géneros } & 1.78 \pm 0.09 & 1.81 \pm 0.12 & 2.03 & 0.24 & 0.81 \\ & \text { Familias } & & 1.91 \pm 0.09 & 7.59 & 1.02 & 0.31 \\ \text { J' } & \text { Géneros } & 0.66 \pm 0.03 & 0.66 \pm 0.04 & 0.42 & 0.06 & 0.95 \\ & \text { Familias } & & 0.69 \pm 0.02 & 4.02 & 0.75 & 0.46 \\ \Delta^{*} & \text { Géneros } & 72.93 \pm 0.41 & 72.65 \pm 0.25 & 0.38 & 0.58 & 0.56 \\ & \text { Familias } & & 73.14 \pm 0.38 & 0.27 & 0.36 & 0.72\end{array}$


Abundancias agrupadas a nivel género
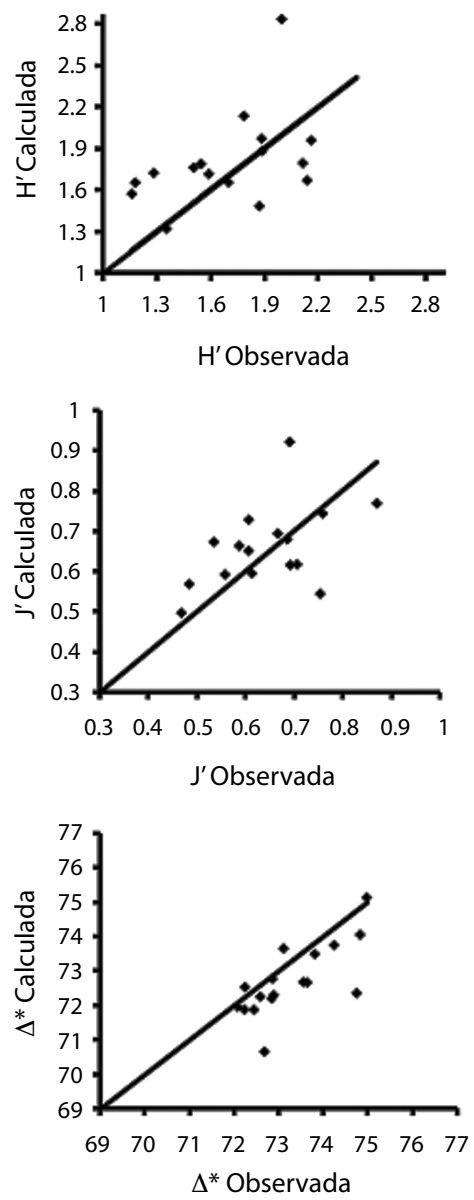

Abundancias agrupadas a nivel familia
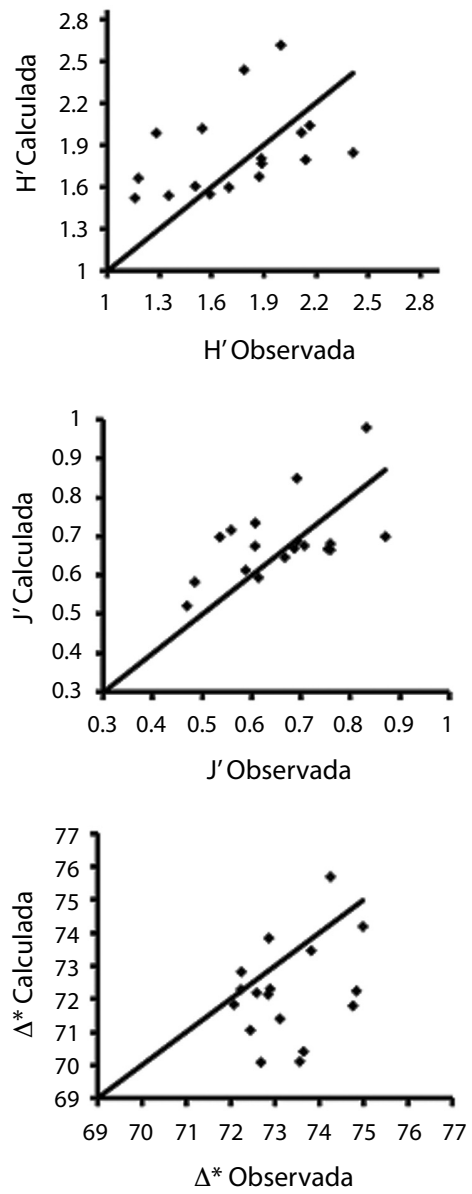

Fig. 2. Relación entre los valores observados en el campo para diversos índices ecológicos (H', J' y $\left.\Delta^{*}\right)$ y las predicciones de los modelos usando géneros y familias indicadoras como factores.

Fig. 2. Relationship between the observed values of the índices (H', J' y $\left.\Delta^{*}\right)$ in the field, and the model predictions using indicator genera and familes.

obstante, vale la pena indicar que el porcentaje de diferencia entre medias, observada y calculada, fue de apenas $0.38 \%$ (Fig. 2).

\section{DISCUSIÓN}

La finalidad de este trabajo fue analizar la factibilidad de predicción de índices ecológicos a partir de la abundancia en niveles taxonómicos superiores, bajo la premisa de que estos indicadores funcionan como marcadores de la condición del sistema, se siguió la línea de los programas de monitoreo de todo el mundo (McField \& Kramer 2007). Se ha discutido que los índices de Shannon $\left(\mathrm{H}^{\prime}\right)$ y Pielou $\left(\mathrm{J}^{\prime}\right)$ son útiles para hacer seguimiento del estado de conservación de los ecosistemas, porque toman en cuenta el número total de especies 
y la homogeneidad con la que se distribuyen sus abundancias. Ambos componentes de la estructura comunitaria han sido interpretados como trasfondo de importantes procesos ecológicos (Magurran 2003). Se deduce que cuando la diversidad de especies es alta, existe una comunidad compleja donde se da un mayor número de interacciones (Magurran 2003) o se considera como más estable (McCann 2000, Jorgensen \& Muller 2000). Estas consideraciones han llevado a aplicar los índices de diversidad como reflejo del estado de conservación de un ecosistema bajo la óptica que éste último mejora conforme aumenta la diversidad, independientemente de cómo sea medida (UNESCO 2003, Mac Nally \& Fleishman 2004, Spellerberg 2005).

En cuanto al índice de distintividad taxonómica $\left(\Delta^{*}\right)$, existe evidencia de que en comunidades dañadas se estrecha el número de grupos taxonómicos mayores, mientras que en condiciones de menor perturbación las especies presentes pertenecen a un número más amplio de taxa superiores (Brown et al. 2002, Koutrakis et al. 2005). Todo ello vuelve atractivo el uso de este índice en un programa de monitoreo, tanto para describir la diversidad biológica en diferentes zonas, o en un área al paso del tiempo, con base en las variaciones del número de grupos taxonómicos presentes. Además $\Delta^{*}$ tiene la ventaja de ser una medida independiente del tamaño de muestra así como del esfuerzo de muestreo (Clarke \& Warwick 1998).

Las diferencias entre épocas de muestreo que son reportadas en este estudio son parte de variaciones similares en todo el suroeste del Golfo de California (Pérez-España et al. 1996, Aburto-Oropeza \& Balart 2001, ArreolaRobles \& Elorduy-Garay 2002), por lo que la inclusión de información proveniente de épocas cálidas y frías en los modelos de regresión se tradujo en predicciones más robustas, pues la variabilidad estacional fue contemplada desde la construcción de cada modelo. Según lo mencionado anteriormente, se puede explicar que las predicciones no fueron afectadas por las diferencias en los índices ecológicos entre épocas de muestreo (Fig. 1). Aunque, los modelos de regresión fueron construidos con datos de abundancia tomados en tres épocas cálidas (Octubre de 2003, 2004 y 2005) y sólo una época fría (Febrero de 2004), la validación de los taxa indicadores se hizo con datos de abundancia tomados durante una época fría (mayo 2006) y aún así, todas las predicciones fueron significativas y se obtuvieron diferencias de medias (observada-calculada) menores al 8\% (Cuadro 8).

Para seleccionar un modelo de predicción de variables indicadoras del estado de un ecosistema, podemos utilizar distintos criterios que respondan a las necesidades de monitoreo (Almada-Villela et al., 2003). En el presente estudio se eligieron tres criterios cuantitativos: un coeficiente de determinación alto, el uso de un bajo número de variables seleccionadas para la predicción y un mínimo porcentaje de diferencia entre el valor medio observado y el calculado por el modelo para cada indicador ecológico.

El coeficiente de determinación es un criterio ampliamente utilizado en la selección de variables indicadoras para programas de monitoreo y en la evaluación de impactos ambientales (Kapustka et al. 2004). Se utiliza como una medida sintética para juzgar el ajuste del modelo de regresión, sin embargo, un coeficiente de determinación alto no necesariamente significa que los datos han sido bien ajustados en la recta, ya que $\mathrm{R}^{2}$ es afectado por la cantidad de información que se introduce al análisis (Tabachnick \& Fidell 1996). Los coeficientes de determinación altos reflejan la exactitud en el ajuste para un conjunto de datos determinado, sin embargo no hay garantía que la precisión en predicciones futuras sea tan eficiente (Chaterjee \& Price 1977, Tabachnick \& Fidell 1996); eso debe ser determinado con análisis ad hoc como el efectuado aquí (Cuadro 8).

El número de variables seleccionadas para la predicción, se enfoca a las limitantes de financiamiento, tiempo, personal y entrenamiento que enfrentan los manejadores de áreas protegidas. De ahí se infiere la importancia de ponderar el potencial de predicción de un modelo contra la cantidad de bioindicadores 
que requieren ser cuantificados. En esta investigación se sugiere que el mayor número de variables a utilizar para el monitoreo sea de 20 a nivel género ( $41 \%$ del total censado), mientras que el menor puede considerar nueve variables a nivel familia (36\% del total censado; Cuadro 1). La mayoría de los modelos aquí propuestos (Cuadro 1) representan una notable disminución de esfuerzo durante la toma de información en campo, ya que en general se requiere censar aproximadamente la mitad de los géneros o especies para llegar a predicciones excelentes de los valores de $\mathrm{H}^{\prime}, \mathrm{J}^{\prime}$ y $\Delta^{*}$, que hayan sido calculados de la forma típica, empleando el total de información a nivel especie (Cuadro 1).

Por último, el porcentaje de diferencia entre el promedio observado y calculado de los valores de los índices ecológicos fue mínimo (siempre $<10 \%$, Cuadro 8 ), e indica que se puede hacer una eficiente evaluación del estado general de la comunidad íctica con una serie reducida y simplificada de datos tomados en el campo; sin embargo no debe olvidarse que puede no ser muy precisa para sitios específicos, dada la ocurrencia de cardúmenes en movimiento que afectan los cálculos. Los modelos estadísticos aquí sugeridos, permiten una evaluación general rápida y además pueden facilitar la comunicación de los resultados del monitoreo para público no especializado, pues permite expresar los cambios del sistema entre un estado y otro en unidades relativas (porcentaje). Esto último representa una ventaja para el manejo de un área natural protegida, dadas las necesidades de gestión y comunicación al exterior (UNESCO 2003), ya que el público y cualquier personal no especializado (incluyendo políticos y otros tomadores de decisiones) requieren de información lo más simplificada posible para realizar sus labores o para informarse de la situación de un sitio o ecosistema. Por ejemplo, el encargado del área podría reportar variaciones en la diversidad como un aumento/disminución del $5 \%$ respecto al año anterior, lo cual es simple de entender para cualquier tipo de público, aún sin preparación en el ámbito de las ciencias biológicas.
El presente estudio demostró la factibilidad de usar indicadores elegidos in situ, hecho que promete mejores resultados en contraste con la importación de técnicas que muchas veces son aplicadas sin probar primero su verdadera utilidad local. En relación con las especies clave para los modelos de predicción (Cuadros 2-5), destacaron el lábrido arcoíris Thalassoma lucasanum (Gill, 1862) y la damisela Chromis atrilobata (Gill, 1862). Estos peces son relevantes en la comunidad de peces del arrecife de Cabo Pulmo porque aportan más del $62 \%$ de la abundancia total, con una frecuencia de ocurrencia de hasta el $97.2 \%$ para el lábrido y $72.2 \%$ para el pomacéntrido; esta generalidad fue registrada también por Villarreal-Cavazos et al. (2000) y Álvarez-Filip et al. (2006) y también ambas especies se reconocen como dominantes en todo el sur del Golfo de California (Pérez-España et al. 1996, Aburto-Oropeza \& Balart 2001). La recurrente presencia de estos géneros en los modelos de regresión, es evidencia de su influencia sobre la diversidad local, lo que a su vez les hace muy eficientes como variables predictivas de las condiciones en el arrecife.

La alta dominancia en la comunidad íctica de Cabo Pulmo también podría implicar el cálculo sesgado de los índices ecológicos, pero la exclusión de especies tan numerosas como Thalassoma lucasanum y Chromis atrilobata, impediría la comparación con registros históricos en los que sí han sido incluidas (Reyes-Bonilla \& Álvarez-Filip 2008). Además, al excluir especies dominantes también podría resultar contraproducente al tratar de interpretar ecológicamente los cambios en los índices comunitarios, ya que su elevada abundancia también es un reflejo de su importancia ecológica en la localidad (Siqueiros-Beltrones 2005). Por ello, la dominancia debe considerarse una propiedad natural de la ictiofauna de ecosistemas arrecifales, de tal forma que la eliminación de las especies con grandes abundancias no tiene sentido bajo la óptica ecológica, aunque quizá si en la estadística.

Existen numerosos estudios que destacan la importancia ecológica de las especies raras 
(Cao et al. 1998, 2001), aunque se desconoce su efecto sobre la diversidad del arrecife de Cabo Pulmo. Debido a su baja abundancia, las especies raras difícilmente son seleccionadas como variables predictivas en los modelos de regresión, por lo que el método aquí propuesto resulta poco sensible a los cambios en este tipo de especies. No obstante, ello no impidió obtener buenas predicciones de los índices comunitarios (Cuadro 8).

Otros géneros, como Kyphosus, Plagiotremus y Abudefduf son típicos de zonas someras y de interfase arena a roca (Thomson et al. 2000), también resultaron seleccionados como buenos predictores en los modelos de regresión, a pesar de haber presentado baja abundancia $(<1 \%)$ y ocurrencia $(<25 \%)$ en los años 2003-2006 (Álvarez-Filip et al. 2006). En este caso podría tratarse de que la rareza del grupo mejora el ajuste del modelo en relación con sitios específicos dentro del arrecife y ello conduce a la inclusión de dichos factores (Neter et al. 1996). En resumen, ciertos géneros o familias indicadoras podrían no ser tan relevantes ecológicamente, pero resultan fundamentales para la predicción estadística de la diversidad (Kolchin et al. 2002).

Es interesante observar que las familias que más aportan a la varianza explicada para la predicción de $\Delta^{*}$, son depredadores u omnívoros que en su mayoría incluyen especies de importancia comercial y que fueron pescadas antes del establecimiento del área protegida. Un ejemplo son las familias Lutjanidae, Carangidae, Serranidae y Labridae (Cuadro 7), cuyo efecto general sobre la diversidad taxonómica $\left(\Delta^{*}\right)$ es positivo; es decir, donde hay especies de alto nivel trófico, habrá mayor diversidad y una mejor repartición de las abundancias relativas en niveles taxonómicos mayores (Clarke \& Warwick 1998). Tal hallazgo apunta a que el efecto de la protección que ha recibido el parque (Arizpe-Covarrubias 2008) ha favorecido la complejidad estructural de la ictiofauna a escala local, aunque es interesante anotar que los carnívoros han causado una baja en la diversidad general del ecosistema arrecifal en la última década (Reyes-Bonilla \& Álvarez-Filip 2008).
Es oportuno mencionar que en el año 2005 se arrancó un programa de capacitación y monitoreo formal del arrecife de Cabo Pulmo que es conducido por una Organización No Gubernamental apoyada en combinación con las autoridades del parque y los residentes locales. En este programa, los responsables del monitoreo deben estar capacitados para identificar 69 especies de peces y 40 especies de invertebrados (similar a un muestreo científico en la localidad), además de saber aplicar bajo el agua las metodologías típicas que son utilizadas para este fin como transectos de banda, cilindros de observación y otros (F. FernándezRivera Melo, com. pers.). La información biológica resultante es muy valiosa, pero existen dos problemas a considerar; a) la complejidad del procedimiento, que implica gran conocimiento por parte de los censadores (e.g. cantidad de especies a identificar) y b) que los recursos requeridos en preparar al personal y llevar a cabo el muestreo no son federales, sino que son otorgados por organizaciones civiles, es decir, estos pueden agotarse eventualmente y ello pone en riesgo la sostenibilidad del esfuerzo a largo plazo y la eficiencia de las acciones de manejo.

Bajo esta perspectiva, el presente trabajo ofrece un antecedente científico para apoyar los esfuerzos de monitoreo, en este caso mediante el uso de bioindicadores propios de la localidad y técnicas cuyo éxito ha sido probado estadísticamente. El uso de los datos a nivel género y familia significan una reducción sustancial en el nivel de entrenamiento que se requeriría para preparar a los responsables del monitoreo, así como de la cantidad de tiempo, dinero y personal dedicados a la recolecta de datos en campo. La información arrojada por el método es altamente confiable porque reconoce el estado general de la ictiofauna del arrecife y ayuda a efectuar estimaciones económicas y altamente eficientes.

\section{AGRADECIMIENTOS}

Cabo Pulmo Divers, Lorenzo Álvarez (University of Sheffield) y estudiantes del 
Laboratorio de Sistemas Arrecifales (UABCS) ofrecieron apoyo para la toma de datos en campo y Saúl González (UABCS) apoyó en la edición del documento. Gabriela Anaya (NIPARAJA A.C.) y Francisco FernándezRivera Melo (COBI A.C.) ofrecieron valiosos comentarios para la consolidación del estudio. La Comisión Nacional de Áreas Naturales Protegidas ofreció facilidades y permisos para la realización del trabajo de campo y el financiamiento para la recolecta de datos provino de CONACYT (Proyecto 37528-B, a L.E. Calderón Aguilera, CICESE, Ensenada) y las fundaciones PADI Aware y Sigma Xi (a L. Alvarez). A Gustavo De la Cruz por sus comentarios para mejorar este manuscrito.

\section{RESUMEN}

El uso de indicadores biológicos es una herramienta útil para la descripción de las comunidades y permite ahorrar recursos en programas de monitoreo. Con el fin de evaluar el potencial bioindicador de grupos taxonómicos superiores en el Parque Nacional Cabo Pulmo, México, se construyeron modelos de regresión múltiple por pasos con valores de abundancia agrupados a nivel género y familia como predictores, mediante el uso de variables dependientes, los índices ecológicos de diversidad (H'), equidad (J') y distintividad taxonómica $\left(\Delta^{*}\right)$. Posteriormente se compararon los resultados de los modelos contra los valores de los índices ecológicos observados en un muestreo independiente. Los resultados mostraron que todos los modelos fueron altamente significativos; los valores más altos del coeficiente de determinación se obtuvieron en las regresiones aplicadas a $\mathrm{H}^{\prime}$, mientras que las usadas por $\Delta^{*}$ fueron las menos precisas. Los resultados sugieren que los modelos predictivos aquí generados son susceptibles de ser aplicados en un programa de monitoreo. Este estudio sugiere la implementación de un programa de monitoreo a largo plazo basado en bioindicadores de la estructura comunitaria de peces en el Parque Nacional Cabo Pulmo, el cual combine la precisión con la facilidad de obtención de datos.

Palabras clave: Golfo de California, índices ecológicos, peces arrecifales, monitoreo de arrecifes, manejo de áreas marinas, suficiencia taxonómica.

\section{REFERENCIAS}

Aburto-Oropeza, O. \& E.F. Balart. 2001. Community structure of reef fish in several hábitats of rocky reef in the Gulf of California. Mar. Ecol. 22: 283-305.
Almada-Villela, P.C., P.F. Sale, G. Gold-Bouchot \& B. Kjerfve. 2003. Manual de métodos para el programa de monitoreo sinóptico del SAM. Proyecto para el Sistema Arrecifal Mesoamericano (SAM). Coastal Resources Multi-Complex Building, Ciudad de Belice, Belice.

Álvarez-Filip, L. \& H. Reyes-Bonilla. 2006. Comparison of community structure and functional diversity of fishes at Cabo Pulmo coral reef, Western Mexico between 1987 and 2003. Proc. 10th Int. Coral Reef Symp. 216-225. Okinawa, Japón.

Álvarez-Filip, L., H. Reyes-Bonilla \& L.E. CalderónAguilera. 2006. Community structure of fishes in Cabo Pulmo Reef, Gulf of California. Mar. Ecol. 27: 253-262.

Arreola-Robles, J.L. \& J.F. Elorduy-Garay. 2002. Reef fish diversity in the region of La Paz, Baja California Sur, Mexico. Bull. Mar. Sci. 70: 1-18.

Arizpe-Covarrubias, O. 2004. El turismo como alternativa a la pesca en el manejo de un arrecife coralino. Cabo Pulmo, Golfo de California, p. 573-588. In E. RiveraArriaga, G.J. Villalobos-Zapata, I. Azuz-Adeath \& F. Rosado-May (eds.). 2004. El Manejo Costero en México. Universidad Autónoma de Campeche, SEMARNAT CETYS-Universidad, Universidad de Quintana Roo, Quintana Roo, México.

Arizpe-Covarrubias, O. 2008. Caracterización del arrecife coralino de Cabo Pulmo, p. 53-74. In A.E. Gámez (ed.). 2008. Turismo y sustentabilidad en Cabo Pulmo, B.C.S. SDSU, UABCS, CONACYT, Baja California Sur, México.

Birkeland, C. 1997. Life and Death of Coral Reefs. Chapman \& Hall, Nueva York, EEUU.

Bohnsack, J.A. \& S.P. Bannerot. 1986. A stationary visual census technique for quantitatively assessing community structure of coral reef fishes. NOAA Technical Report NMFS, 41. NOAA/SSFC, Florida, EEUU.

Brown, B.E., K.R. Clarke \& R.M. Warwick. 2002. Serial patterns of biodiversity change in corals across shallow reef flats in Ko Phuket, Thailand, due to the effects of local (sedimentation) and regional (climatic) perturbations. Mar. Biol. 141: 21-29.

Brusca, R.C. \& D.A. Thomson. 1975. Pulmo reef: the only "coral reef" in the Gulf of California. Cien. Mar. 1: 37-53.

Cao, Y., D.D. Williams \& N.E. Williams. 1998. How important are rare species in aquatic community ecology and bioassessment? Limnol. Oceanogr. 43: 1403-1409. 
Cao, Y., D.P. Larsen \& R.S.J. Thorne. 2001. Rare species in multivariate analysis for bioassessment: some considerations. J.N. Am. Benthol. Soc. 20: 144-153.

Chaterjee, S. \& B. Price. 1977. Regression analysis by example. Wiley, Nueva York, EEUU.

Clarke, K.R. \& R.M. Warwick. 1998. A taxonomic distinctness index and its statistical properties. J. Appl. Ecol. 35: 523-531.

Clarke, K.R. \& R.M. Warwick. 2001 Change in marine communities. Primer-E, Plymouth, Inglaterra.

CONANP. 2006. Programa de Conservación y Manejo del Parque Nacional Cabo Pulmo. Comisión Nacional de Áreas Naturales Protegidas, México D.F., México.

Diario Oficial de la Federación (DOF). 1995. Decreto por el que se declara área natural protegida con el carácter de Parque Marino Nacional, la zona conocida como Cabo Pulmo, Baja California Sur, México.

Gámez, A.E. 2008. Turismo y sustentabilidad en Cabo Pulmo, B.C.S. SDSU, UABCS, CONACYT, La Paz, Baja California Sur, México.

Jorgensen, S.E. \& F. Muller. 2000. Handbook of ecosystem theories and management. Lewis, Washington, EEUU.

Kapustka, L., G. Biddinger, M. Luxon \& H. Galbraith. 2004. Landscape ecology and wildlife habitat evaluation: Critical information for risk, assessment, land-use management activities, and biodiversity enhancement practices. ASTM, Pensilvania, EEUU.

Kolchin, V.F., V.Y. Kozlov, V.V. Mazalov, Y.L. Pavlov \& Y.V. Prokhorov. 2002. Probabilistic methods in discrete mathematics. Proc. $5^{\text {th }}$ Int. Petrozavodsk Conference, Petrazavodsk, Russia.

Koutrakis, E.T., A.C. Tsikliras \& A.I. Sinis. 2005. Temporal variability of the ichthyofauna in a northern Aegean coastal lagoon (Greece). Influence of environmental factors. Hydrobiologia 543: 245-257.

Ley General del Equilibrio Ecológico y la Protección al Ambiente (LEGEEPA). 2005 año diferente. Diario Oficial de la Federación. México, D.F. México.

Magurran, M. 2003. Measuring biological diversity. Blackwell, Malden, EEUU.

Martínez de la Torre, J.A. 2008. Desarrollo local y el estado de la economía base en Cabo Pulmo, p. 133-162. In A.E. Gámez (ed.). Turismo y sustentabilidad en Cabo Pulmo, B.C.S. SDSU, UABCS, CONACYT. B.C.S. México.
McCann, K.S. 2000. The diversity-stability debate. Nature 405: 228-233.

McField, M. \& P. Kramer. 2007. Healthy reefs for healthy people: A guide to indicators of reef health and social well-being in the Mesoamerican Reef Region. Smithsonian Institution, Washington, D.C., EEUU.

Mac Nally, R. \& E. Fleishman. 2004. A successful predictive model of species richness bases on indicator species. Conserv. Biol. 18: 646-654.

Mograbi, J. \& C.M. Rogerson. 2007. Maximising the local pro-poor impacts of dive tourism: Bahía de Sodwana, Sudafrica. Urban Forum 18: 85-104.

Neter, J., M. Kutner, C. Nachtsheim \& W. Wassermann. 1996. Applied linear estatistical models. McGrawHill, Chigago, EEUU.

Noss, R.F., M.A. O'Conell \& D.D. Murphy. 1997. The science of conservation planning. Island, Washington, D.C., EEUU.

Pérez-España, H., F. Galván-Magaña \& L.A. AbitiaCárdenas. 1996. Variaciones temporales y espaciales en la estructura de la comunidad de peces arrecifales rocosos del suroeste del Golfo de California, México. Cien. Mar. 22: 273-294.

Reyes-Bonilla, H. \& V.H. Beltrán-Ramírez. 1998. Valor de conservación de dos arrecifes del Golfo de California. Comunidades Ictiológicas. Insulario 9-10: 2-8.

Reyes-Bonilla, H. \& L. Álvarez-Filip. 2008. Long-term changes in taxonomic distinctness and trophic structure of the reef fishes at Cabo Pulmo Reef, Gulf of California. Proc. 11th Coral Reef Int. Symp. 790-794. Fort Lauderdale, Florida, EEUU.

Riegl, B.M., J. Halfar, S.J. Purkis \& L. Godinez-Orta. 2007. Sedimentary facies of the Pacific's northern most reef-like setting (Cabo Pulmo, Mexico). Mar. Geol. 236: 61-77.

Robinson, J. \& A. Thomson. 1992. Status of the Pulmo Coral Reefs in the lower Gulf of California. Environ. Conserv. 19: 261-264.

Siqueiros-Beltrones, D.A. 2005. Una paradoja sobre uniformidad Vs. orden y estabilidad en la medida de la diversidad de Especies según la teoría de la información. Ludus Vitalis 24: 83-92.

Spellerberg, I.F. 2005. Monitoring Ecological Change. Cambridge, Cambridge, Inglaterra.

Tabachnick, B.G. \& L.S. Fidell. 1996. Using multivariate statistics. Harper Collins, Nueva York, EEUU. 
Thomson, D.A., L.T. Findley \& A.N. Kerstitch. 2000. Reef fishes of the Sea of Cortez. Wiley, Nueva York, EEUU.

Tilmant, J.T. 1987. Impacts of Recreational Activities on Coral Reefs, p. 197-214. In B. Salvat (ed.). Human Impacts on Coral Reefs: Facts and Recommendations. Antenne Museum E.P.H.E., French Polynesia.

UNESCO. 2003. A reference guide on the use of indicators for integrated Coastal Management. ICAM Dossier, Manuals and Guides, Paris, Francia.
Villareal-Cavazos, A., H. Reyes-Bonilla, B. BermúdezAlmada \& O. Arizpe-Covarrubias. 2000. Los peces del arrecife de Cabo Pulmo, Golfo de California, México: Lista sistemática y aspectos de abundancia y biogeografía. Rev. Biol. Trop. 48: 413-424.

Wilkinson, C. 2002. Status of Coral Reefs of the World. Aust. Inst. Mar. Sci. Australia.

Zar, J.H. 1999. Biostatistical Analysis. Prentice Hall, Englewood Cliffs, Nueva Jersey, EEUU. 